\title{
Sentinel Lymph Node Not Identified
}

National Cancer Institute

\section{Source}

National Cancer Institute. Sentinel Lymph Node Not Identified. NCI Thesaurus. Code C137832.

An indication that sentinel lymph node mapping was attempted but that it did not identify a sentinel lymph node. 